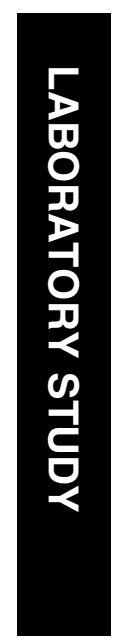

\title{
A structural investigation of corneal graft failure in suspected recurrent keratoconus
}

${ }^{1}$ Structural Biophysics Research Group, School of Optometry and Vision Sciences, Cardiff University, Cardiff, UK

${ }^{2}$ Department of Ophthalmology, Royal Glamorgan Hospital, Llantrisant, UK

\section{${ }^{3}$ Department of} Ophthalmology, Great Wall Hospital of PLA, Beijing, China

Correspondence: KM Meek, School of Optometry and Vision Sciences,

Cardiff University, Maindy Road,

Cathays,

Cardiff, Wales CF24 4LU, UK

Tel: + 44 (0)2920 876317;

Fax: + 44 (0)2920 874859

E-mail: HAYESS5@

CF.AC.UK

Received: 4 February 2009 Accepted in revised form: 29 May 2009

Published online: 3 July 2009

This work has been presented in part at the 2008 British Society for Matrix Biology conference and the 2009 Association for Research in Vision and Ophthalmology conference

\begin{abstract}
Purpose Penetrating keratoplasty was performed on the right eye of a 51-year-old patient diagnosed with advanced bilateral keratoconus. Thirteen years later, an $8.5 \mathrm{~mm}$ regraft was required as a result of gross vascularisation, a poor epithelium, and suspected recurrent keratoconus. To learn more about the structural basis for graft failure, we examined the removed tissue for the presence of abnormalities in the stroma and limiting membranes.

Methods X-ray scattering and electron microscopy were used to study the failed-graft tissue to provide information on the integrity of the limiting membranes and the diameter, dominant orientation, and distribution of collagen within the corneal stroma. The findings were compared with similar structural data from normal and keratoconus corneas.

Results In contrast to the keratoconus cornea, a normal orientation and distribution of collagen was seen throughout most of the failed-graft tissue, although abnormalities were observed in the infero-nasal quadrant at the original graft/ host junction. The average diameter of collagen fibrils in the failed-graft button did not differ from that of normal corneas.

Conclusions The structural abnormalities identified in this case of graft failure were not consistent with those typically seen in keratoconus. The clear demarcation of the graft/ host boundary 13 years after surgery suggests that a normal stromal collagen arrangement may never be fully achieved in corneal graft wounds. Eye (2010) 24, 728-734; doi:10.1038/eye.2009.159; published online 3 July 2009
\end{abstract}

Keywords: recurrent keratoconus; graft failure; cornea; X-ray scattering; collagen
S Hayes ${ }^{1}$, R Young', C Boote', N Hawksworth², $Y$ Huang $^{3}$ and KM Meek'

\section{Introduction}

Keratoconus, a condition characterised by progressive corneal thinning and steepening, and severe irregular astigmatism, is one of the leading causes of corneal transplant surgery. ${ }^{1-3}$ Despite high success rates for penetrating keratoplasty (PK) for keratoconus, which have been calculated to be $98.9 \%$ at 46 months followup $^{4}$ and $93.7 \%$ at 13.8 years, ${ }^{5}$ complications such as graft failure and keratoconus recurrence occur in a small minority of cases. ${ }^{5-9}$

Previous histopathological studies on recurrent keratoconus have shown the presence of tissue abnormalities consistent with keratoconus, such as breaks in the epithelial basement membrane and Bowman's layer, abnormal keratocytes, and stromal thinning. ${ }^{10,11}$ However, as is the case with keratoconus itself, the cause of keratoconus recurrence, which may occur up to 40 years after successful keratoplasty ${ }^{8}$ remains unclear.

The mechanism of graft failure following PK for keratoconus is better understood. It has been documented to occur in $6.3 \%$ of keratoconus patients at a mean time of 12.6 years after surgery and most commonly results from immunological factors or a failure of the endothelium. ${ }^{5}$

Here, we have used X-ray scattering and electron microscopy to examine the stromal ultrastructure of a failed corneal graft with suspected recurrent keratoconus to learn more about the role of collagen in graft failure. Our findings are compared with similar structural data obtained from normal and keratoconus corneas.

\section{Materials and methods}

\section{Specimen information}

A 51-year-old patient with an uncorrected visual acuity of $6 / 60$, which improved to $6 / 18$ 
with pinhole, was diagnosed with advanced bilateral keratoconus in 1993. PK was performed on the right eye (using a 7.5-mm donor button and a $7.25-\mathrm{mm}$ recipient bed) and 9 years later deep lamellar keratoplasty was used to treat the left eye. After PK of the right eye, the patient's corrected visual acuity improved to $6 / 24$ and after treatment of the left eye the patient showed 6/36 (right eye) and 6/18 (left eye) potential with diagnostic contact lens fitting. After application of scleral lenses, visual acuity improved to $6 / 24+$ in the right eye and $6 / 12$ in the left eye.

However, 13 years after the original graft an $8.5-\mathrm{mm}$ regraft was required in the right eye as a result of a poor tear film, multiple punctuate erosions, epitheliopathy, corneal ectasia, gross peripheral vascularisation in the infero-nasal quadrant, oedema in the lower half of the cornea, and suspected recurrent keratoconus. Unfortunately, because of gross tissue distortion and severe epitheliopathy, it was not possible to obtain corneal topography using an EyeSys 3000 Corneal Analysis System (EyeSys Laboratories, Houston, Texas, USA).

Immediately after regraft surgery, the removed 8-mm corneal button was wrapped in Clingfilm (Superdrug stores, Croyden, UK) to prevent tissue dehydration, snap frozen in liquid nitrogen-cooled isopentane, and stored at $-80^{\circ} \mathrm{C}$ until required for data collection. From hereon, this tissue will be referred to as the 'failed-graft' cornea. Maps of collagen orientation and distribution in the failed-graft tissue were compared with those from: (i) a normal 77-year-old right cornea (fixed in $4 \%$ paraformaldehyde), obtained from the Great Wall Hospital (Beijing, China); and (ii) a left 8.0-mm keratoconus corneal button with a history of corneal hydrops 15 months earlier (stored at $-80^{\circ} \mathrm{C}$ ), obtained from a 24-year-old female at the time of PK (Moorfields Eye Hospital, London, UK). The findings were compared with our previously published work (using the same experimental protocol as here), which examined six normal corneas ${ }^{12}$ and six keratoconus corneas. $^{13,14}$

Measurements of fibril diameter in the failed-graft corneal tissue were compared with those obtained from an 8.5-mm button excised from a normal post-mortem cornea stored for 23 days in culture medium and then de-swelled for 2 days in dextran (obtained from the Corneal Transplant Service Eye Bank, Bristol, UK). Our previously published results from five healthy corneas ${ }^{15}$ were used to confirm the normal range of fibril diameters in the human cornea.

We certify that all applicable institutional and governmental regulations concerning the ethical use of human tissue were followed during this research.

\section{$X$-ray scattering data collection and analysis}

X-ray scattering patterns were collected at the UK Synchrotron Radiation Source in Daresbury.

\section{Low-angle X-ray scattering}

Using an X-ray beam of wavelength $0.1544 \mathrm{~nm}$ focussed to measure $1 \times 1 \mathrm{~mm}$ at the sample, $X$-ray scattering patterns were recorded at $1-\mathrm{mm}$ intervals over the normal and failed-graft corneal buttons. The images, each resulting from a 60s (normal cornea) or 90s (failedgraft) X-ray exposure, were collected on a detector placed $6.25 \mathrm{~m}$ behind the specimen. The data were analysed as previously described ${ }^{15}$ to produce measurements of average collagen fibril diameter.

\section{High-angle $X$-ray scattering}

Using a camera length of $150 \mathrm{~mm}$ and an X-ray beam $(0.1488 \mathrm{~nm})$ focussed to measure $0.2 \times 0.2 \mathrm{~mm}$ at the sample, $X$-ray scattering patterns resulting from a $40 \mathrm{~s}$ (failed-graft), $45 \mathrm{~s}$ (normal), or $60 \mathrm{~s}$ (keratoconus) X-ray exposure were recorded at $0.25-\mathrm{mm}$ intervals over the entire keratoconus and failed-graft corneal buttons, and at $0.4 \mathrm{~mm}$ over the normal cornea. The X-ray scatter patterns were normalised against variations in X-ray beam intensity and exposure time and analysed using methods previously described. ${ }^{14}$ For each cornea, the analysed data were assembled to show the relative mass distribution of total fibrillar collagen and that of preferentially aligned collagen (defined as additional $X$-ray scatter from collagen lying in a specific orientation after removal of $X$-ray scatter from collagen disposed in all directions). To show the predominant orientation of collagen at each sampling position, vector coordinates/ plots were formed in which the distance from the centre of the plot in a given direction represents the relative amount of aligned collagen lying in that direction. We have previously shown that changes in tissue hydration do not affect the preferred orientation of collagen in the cornea, however, the intensity of the X-ray scatter does decrease with increased tissue hydration. ${ }^{16}$ As the hydration of the corneas used in this study was not measured (owing to the tissue being processed for electron microscopy), the X-ray scattering intensity shown in the contour maps may not be taken as absolute values. However, comparisons of the relative distribution of collagen within and between individual corneas are valid.

Between X-ray data collections and electron microscopy processing, the failed-graft tissue was immersed in $4 \%$ paraformaldehyde and stored at $4^{\circ} \mathrm{C}$.

\section{Electron microscopy}

Full-thickness tissue blocks measuring $\sim 2 \times 0.5 \mathrm{~mm}$ were cut from the central and peripheral locations of the 
fixed failed-graft corneal button as shown in Figure 1. After a brief rinse in $0.1 \mathrm{M}$ sodium phosphate buffer, they were postfixed, first in $1 \%$ osmium tetroxide for $1 \mathrm{~h}$, then in aqueous $0.5 \%$ uranyl acetate for $1 \mathrm{~h}$, with an interim wash in distilled water. After dehydration in a graded ethanol series, they were infiltrated and embedded in

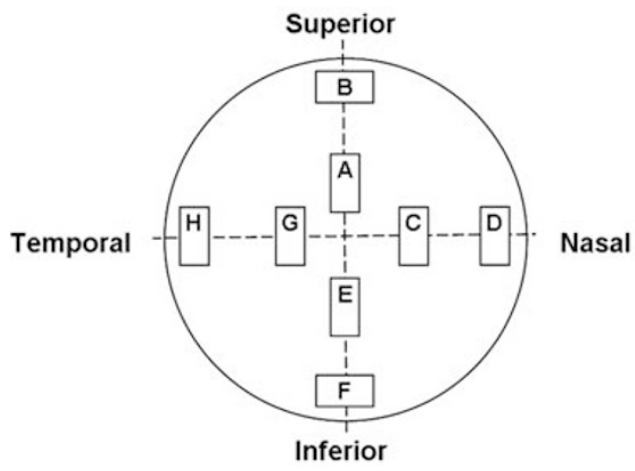

Figure 1 Schematic of the failed-graft corneal button showing the location of ultra-thin sections removed for electron microscopy examination.
Araldite CY212 resin (Agar Scientific Ltd., Stanstead, Essex, UK) and polymerised at $60^{\circ} \mathrm{C}$. Sections of $100 \mathrm{~nm}$ were cut using a Leica UC6 ultramicrotome (Leica Microsystems Ltd, Milton Keynes, UK) stained with saturated uranyl acetate and lead citrate, and examined in a Philips EM208 transmission electron microscope (FEI Company, Eindhoven, Netherlands). A series of images were acquired at magnifications of 4000 and 16000 from the outer, mid, and posterior stromal sites in each of the eight locations shown in Figure 1.

\section{Results}

\section{Fibril diameter}

Individual measurements of fibril diameter recorded from 60 corresponding locations across the normal and failed-graft corneal buttons were averaged and the standard error of the mean calculated. The average fibril diameter and standard error was found to be $33.8 \pm 0.05 \mathrm{~nm}$ in the normal cornea and $34.5 \pm 0.07 \mathrm{~nm}$ in the failed-graft tissue. Although the average fibril

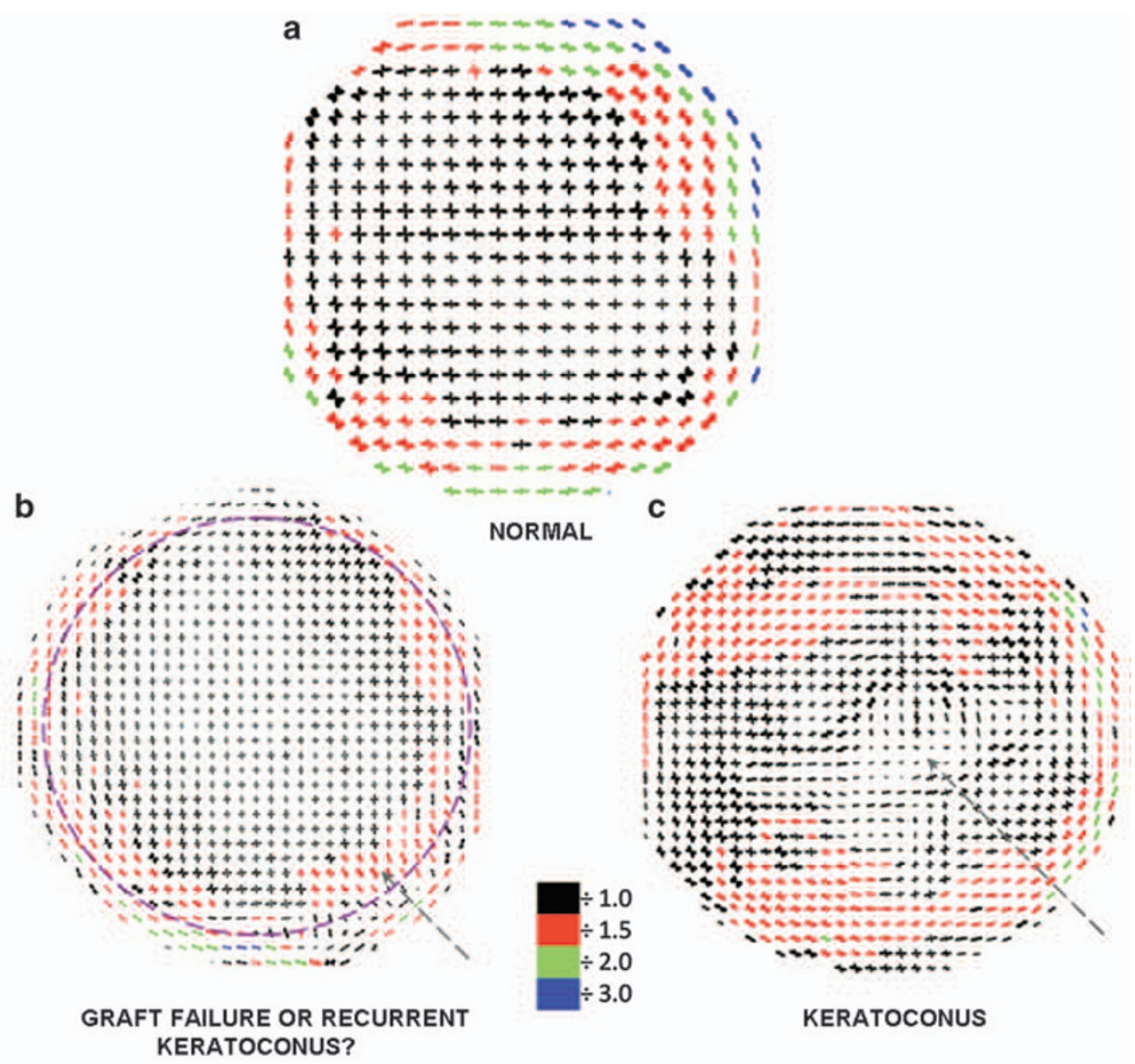

Figure 2 Vector plot maps showing the predominant direction of collagen throughout the central $8 \mathrm{~mm}$ of a normal cornea (measured at 0.4-mm intervals) (a), a failed-graft cornea with suspected recurrent keratoconus (b), and a keratoconus cornea (c) (both measured at $0.25-\mathrm{mm}$ intervals). Owing to the variations in the amount of aligned collagen across the cornea, it was necessary to scale down the larger vector plots by the factors shown in the key. The dashed purple circle in (b) represents the approximate location of the original 7.5-mm graft for keratoconus. Grey arrows in (b) and (c) indicate regions of abnormal collagen orientation. 
a

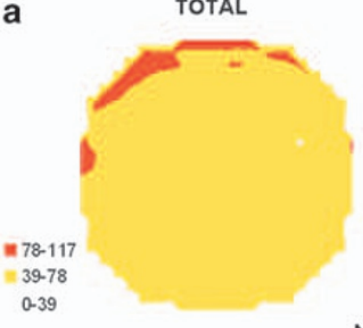

C

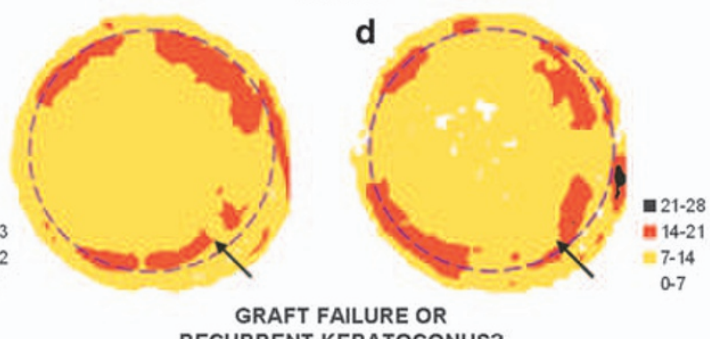

e

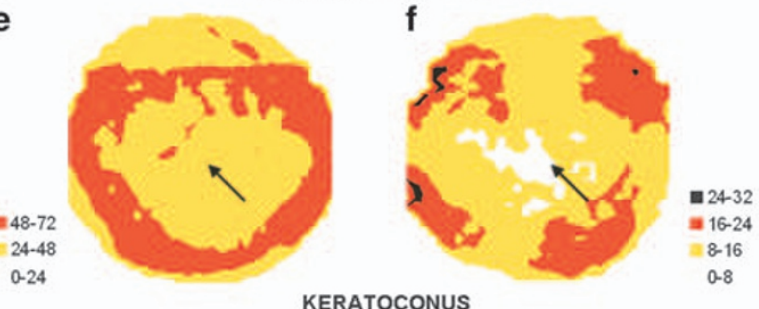

Figure 3 Contour maps showing the relative distribution (based on X-ray scatter intensity) of total collagen and preferentially aligned collagen in the central $8 \mathrm{~mm}$ of a normal cornea ( $a$ and b), a failed-graft cornea with suspected recurrent keratoconus (c and d), and a keratoconus cornea (e and f). Black arrows indicate the location of the most marked disruption to normal collagen orientation as shown in Figure 2.

diameter in the failed-graft button was higher than that of the normal cornea $(P<0.001)$, they were both within the typical range for healthy human corneas. ${ }^{15}$ The variation in fibril diameters across the cornea was consistent for both the normal and failed-graft tissue.

\section{Predominant orientation of corneal collagen}

In the central $6 \mathrm{~mm}$ of the normal cornea, collagen lies predominantly in the superior-inferior and nasaltemporal directions. This is shown in the form of crossshaped vector plots (Figure $2 \mathrm{a}$ ). With increasing distance from the centre, the predominantly orthogonal orientation of collagen becomes gradually obscured by additional collagen aligned tangentially to the corneal edge. This specific arrangement has been well characterised previously in our laboratory. ${ }^{12,17}$

Figure $2 \mathrm{~b}$ shows predominant orientation of collagen in the failed-graft tissue. The approximate position of the original $7.25 \mathrm{~mm}$ PK graft-host interface is highlighted by a purple dashed circle. As seen in the normal cornea, an orthogonal preferred orientation of collagen exists within the central $6 \mathrm{~mm}$ region of the failed-graft button. However, beyond this, abnormalities in collagen orientation were observed in the infero-nasal corneal quadrant at the original graft-host interface (highlighted by a grey arrow). In this region, the normal tangential orientation of collagen is replaced by radially aligned collagen, and it appears that the ectasia extends across the interface and includes both the original keratoconus cornea as well as the grafted cornea. The remaining three quadrants of the failed-graft tissue exhibited normal collagen orientation.

In contrast to the failed-graft tissue, collagen orientation appeared normal beyond the central $6 \mathrm{~mm}$ of the keratoconus button. However, with increasing proximity to the apex of the cone, the normal orthogonal orientation of collagen was gradually lost (Figure 2c). These findings are consistent with our previously published data from six keratoconus corneas of varying disease severity, cone shape, and location. ${ }^{13,14}$

\section{Collagen distribution}

In accordance with our previous findings, ${ }^{12,17}$ a uniform distribution of total collagen was observed throughout most of the central $8 \mathrm{~mm}$ of the normal cornea (Figure 3a). The increase in collagen seen towards the edge of the button coincides with an increase in tissue thickness in the peripheral cornea. In all quadrants of the normal corneal button, an excess of collagen lies tangentially to the edge of the cornea to produce a characteristic skewed diamond-shaped arrangement in the aligned collagen contour map (Figure $3 b$ ). This arrangement has been shown to be highly reproducible in normal corneas and exhibits midline symmetry between the left and right eyes. ${ }^{12}$

As a consequence of stromal swelling in both the keratoconus (due to a prior case of corneal hydrops) and failed-graft corneas, the overall X-ray scatter from these tissues was lower than that of the normal cornea.

However, abnormalities in the relative distribution of collagen mass were observed in both the failed-graft tissue (Figures $3 \mathrm{c}$ and $\mathrm{d}$ ) and keratoconus corneal button (Figures 3e and f). Within the failed-graft button, arcs of increased total collagen scatter (highlighted by a black arrow in Figure 3c) were seen well within the central $8-\mathrm{mm}$ region and coincided with the boundary of the original 7.5-mm corneal graft. The normal skewed diamond-shaped arrangement of aligned collagen was also disturbed in both the failed-graft tissue (Figure $3 \mathrm{~d}$ ) and keratoconus cornea (Figure 3f). Within the failedgraft tissue, regions of high collagen alignment were observed at the original graft-host interface. 
Central cornea

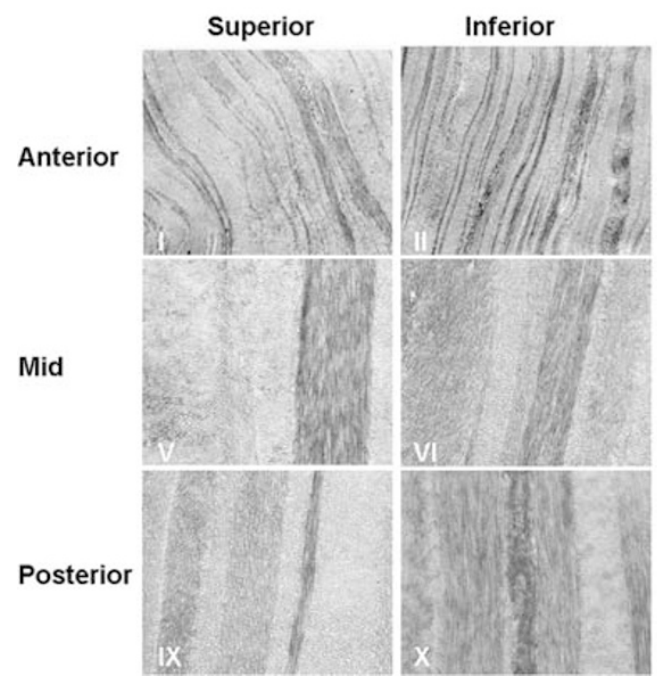

Peripheral cornea

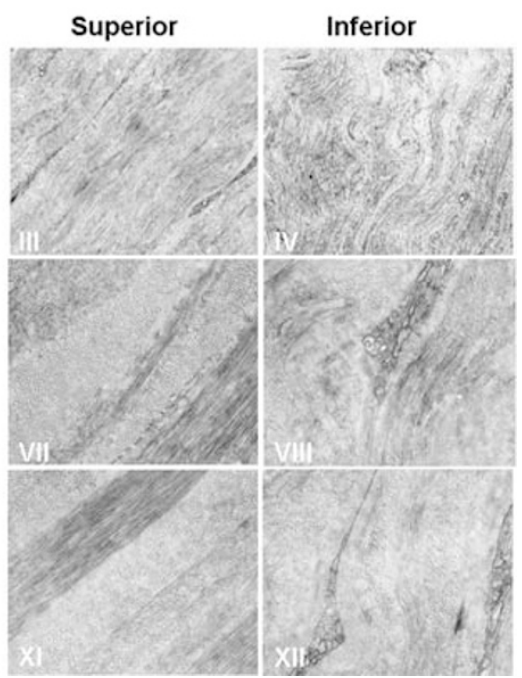

Figure 4 Electron microscopy images from the anterior, mid, and posterior stroma of the superior and inferior aspects of the central and peripheral regions of a failed-graft button. Images were recorded at 4000 (I-IV) and $\times 16000$ (V-XII) magnification. With reference to Figure 1, superior central corneal images (I, V, and IX) correspond to tissue location A, inferior central (II, VI and X) to E, superior peripheral (III, VII and XI) to B, and inferior peripheral (IV, VIII and XII) to F. Gross lamellar disruption is seen at all tissue depths in the inferior aspect of the peripheral cornea.

\section{Electron microscopy findings}

Examples of electron microscopy images recorded from the anterior, mid, and posterior stroma of the superior and inferior regions of the failed-graft tissue (locations $\mathrm{A}$, B, E, and F in Figure 1) are shown in Figure 4. A normal lamellae arrangement was seen at all tissue depths in all quadrants of the central cornea (I, II, V, VI, IX, and X). However, in the inferior aspect of the peripheral cornea, the lamellae were wavy and less easily demarcated than elsewhere in the cornea (IV, VIII, and XII). In this region, lamellar disorder was evident throughout the entire thickness of the tissue. The remainder of the peripheral cornea appeared normal with distinct lamellae present at all tissue depths (III, VII, and XI).

There was no evidence of breaks in Bowman's layer or Descemet's membrane in any region of the cornea.

\section{Discussion}

Despite the high success rate of PK for keratoconus, complications such as allograft rejection, graft failure, and recurrent keratoconus do occasionally occur, although the reasons for these outcomes are not well understood. In this study, we examined the ultrastructure of a failed corneal graft with suspected recurrent keratoconus that presented 13 years after the original PK for keratoconus with a poor tear film, multiple punctuate erosions, epitheliopathy, and gross peripheral vascularisation in the infero-nasal quadrant.
The findings were compared with similar structural data obtained from normal and keratoconus corneas.

Examination of the failed-graft tissue by electron microscopy did not show any corneal changes consistent with a recurrence of keratoconus, such as, breaks in the epithelial basement membrane, fragmentation of Bowman's layer, or the presence of abnormal keratocytes. ${ }^{10,11}$ Although $\mathrm{X}$-ray scattering showed abnormalities in terms of collagen orientation and distribution to exist in both the failed-graft and keratoconus buttons, the specific location and nature of the changes differed between the two tissues. Despite the considerable variability known to exist between keratoconus corneas in terms of the extent of stromal collagen disruption, ${ }^{14} \mathrm{X}$-ray scattering and scanning electron microscopy studies are in agreement that the severity of disruption decreases with distance from the cone apex. ${ }^{13,14,18,19}$ In contrast to most keratoconus corneal buttons, the majority of the failed-graft tissue appeared normal in terms of collagen orientation and mass distribution, with regions of abnormality being limited to the inferior aspect of the peripheral cornea and coinciding in each case with the location of the original graft-host interface. The most marked abnormalities were observed in the grossly vascularised infero-nasal quadrant of the peripheral cornea, where the normal tangential alignment of collagen was replaced by radially orientated collagen and the lamellae appeared indistinct and disordered when viewed by electron microscopy. Lamellar disruption may have occurred during the early wound-healing process, possibly by an unequal tightness 
of sutures around the corneal graft. Alternatively, the structural irregularities observed in the periphery of the donor button may have occurred as a result of an advancement of keratoconus in the residual host cornea and the subsequent infiltration of abnormal collagenproducing keratocytes into the donor tissue. ${ }^{5,20}$ A further possibility is that collagen remodelling may have occurred in the cornea following keratocyte activation in response to the presence of punctuate erosions and epitheliopathy. Unfortunately, only a very narrow rim (about 0.5-mm wide) of the original keratoconus peripheral cornea was present, as the excised button was $<1 \mathrm{~mm}$ wider than the original graft. Owing to the lamellar distortions known to occur at the edge of corneal incisions (unpublished results), it was not possible to examine specifically the keratoconus rim of the excised corneal button using electron microscopy. From the X-ray scattering results (Figure 2) it appears that the inferonasal ectasia extends across the interface and includes both the original keratoconus cornea as well as the grafted cornea. However, the uncertainty in the precise location of the boundary between the original keratoconus tissue and the grafted cornea means that it is not possible to say unambiguously whether the original keratoconus tissue is ectatic. However, it is clear that the lamellar distortion extends into the donor cornea.

Collagen fibril diameter in the affected infero-nasal quadrant of the failed-graft cornea did not differ significantly from that measured elsewhere within the same tissue or from those of other normal and unscarred keratoconus corneas, ${ }^{15,21}$ suggesting that scar tissue (which has a larger fibril diameter) ${ }^{22}$ was not present. However, it must be remembered that X-ray scattering data provide an average measurement of fibril diameters throughout the entire thickness of the cornea at the point through which the beam passes. Therefore, a small change in fibril diameter at a specific tissue depth may not result in a significant change to the average value when measured by this technique.

Although the diagnosis of suspected keratoconus in this failed graft was not confirmed by structural investigation, it is clear that 13 years after surgery, the donor and recipient tissue remain structurally distinct and the junction between the two tissues is marked by a relative increase in collagen mass. The increase in intermolecular X-ray scatter in this region cannot be explained by a higher number of collagen molecules per fibril or by an increase in the density of collagen fibrils, as low-angle X-ray scattering data showed the diameter of the fibrils and spacing between them to be normal. The relative increase in collagen mass at the graft-host interface must therefore be due to the presence of additional corneal tissue. Possible explanations for this may include the deposition of additional newly formed collagen during wound healing, a disparity between the size of the 7.5-mm corneal graft and the 7.25-mm recipient hole, or a slight slippage of the corneal graft after surgery, resulting in graft/host tissue overlap.

The clear demarcation seen here between the graft and recipient corneal tissue, along with reports of wound dehiscence up to 19 years after surgery, ${ }^{23}$ and stress test studies that show the graft-host interface remains weak even after the wound appears to be fully healed, ${ }^{24}$ all support the belief that corneal wound healing occurs mainly in the epithelium and endothelium and that a normal stromal architecture may never fully be achieved in corneal graft wounds.

\section{Acknowledgements}

The authors thank Dr Val Smith (Corneal Transplant Service Eye Bank, Bristol, UK) and Mr Stephen Tuft (Moorfields Eye Hospital, London, UK) for the provision of samples and Drs Mike MacDonald, Gunter Grossman, and Katolitina Geraki (Synchrotron Radiation Source, Daresbury, UK) for their assistance with data collection. This study received programme grant support from the Medical Research Council (Grant G0600755) and the Council for the Central Laboratory of Research Councils. Professor Keith Meek is a Royal Society-Wolfson Research Merit Award Holder. The funding sources had no involvement in the design of this study or in the collection, analysis, and interpretation of data.

\section{References}

1 Cosar C, Sridhar MS, Cohen EJ, Held EL, Alvim Pde T, Rapuano CJ et al. Indications for penetrating keratoplasty and associated procedures, 1996-2000. Cornea 2002; 21: 148-151.

2 Al-Yousuf N, Mavrikakis I, Mavrikakis E, Daya SM. Penetrating keratoplasty: indications over a 10 year period. Br J Ophthalmol 2004; 88: 998-1001.

3 Ghosheh FR, Cremona FA, Rapuano CJ, Cohen EJ, Ayres $\mathrm{BD}$, Hammersmith KM et al. Trends in penetrating keratoplasty in the United States 1980-2005. Int Ophthalmol 2008; 28: 147-153.

4 Lim L, Pesudovs K, Coster DJ. Penetrating keratoplasty for keratoconus: visual outcome and success. Ophthalmol 2000; 107: 1125-1131.

5 Pramanik S, Musch DC, Sutphin JE, Farjo AA. Extended long-term outcomes of penetrating keratoplasty for keratoconus. Ophthalmol 2006; 113: 1633-1638.

6 Abelson MB, Collin HB, Gillette TE, Dohlman CH. Recurrent keratoconus after keratoplasty. Am J Ophthalmol 1980; 90: 672-676.

7 Nirankari VS, Karesh J, Bastion F, Lakhanpal V, Billings E. Recurrence of keratoconus in donor cornea 22 years after successful keratoplasty. Br J Ophthalmol 1983; 67: 23-28. 
8 Thalasselis A, Etchepareborda J. Recurrent keratoconus 40 years after keratoplasty. Ophthal Phys Optics 2002; 22 330-332.

9 Patel SV, Tester R, Soong HK, Sugar A, Farjo QA. Recurrent ectasia after penetrating keratoplasty for keratoconus. Invest Ophthalmol Vis Sci 2005; 46, E-Abstract 4948.

10 Verinder S, Nirankari JK, Bastion F, Lakhanpal V, Billings E. Recurrence of keratoconus in donor cornea 22 years after successful keratoplasty. $\mathrm{Br}$ J Ophthalmol 1983; 67: 23-28.

11 Unal M, Yücel I, Akar Y, Akkoyunlu G, Ustünel I. Recurrence of keratoconus in two corneal grafts after penetrating keratoplasty. Cornea 2007; 26: 362-364.

12 Boote C, Hayes S, Abahussin M, Meek KM. Mapping collagen organisation in the human cornea: left and right eyes are structurally distinct. Invest Ophthalmol Vis Sci 2006; 47: 901-908.

13 Meek KM, Tuft SJ, Huang Y, Gill PS, Hayes S, Newton $\mathrm{RH}$ et al. Changes in collagen fibril orientation in keratoconus corneas. Invest Ophthalmol Vis Sci 2005; 46: 1948-1956.

14 Hayes S, Boote C, Tuft S, Quantock AJ, Meek KM. A study of corneal thickness, shape and collagen organisation in keratoconus using videokeratography and x-ray scattering techniques. Exp Eye Res 2007; 84: 423-434.

15 Boote C, Dennis S, Newton R, Puri H, Meek KM. Collagen fibrils appear more closely packed in the prepupilliary cornea: optical and biomechanical implications. Invest Ophthalmol Vis Sci 2003; 44: 2941-2948.
16 Meek KM, Hayes S, Boote C. The effect of swelling on the lamellar arrangement of the corneal stroma. Invest Ophthalmol Vis Sci 2005; 46, E-Abstract 2183.

17 Aghamohammadzadeh H, Newton RH, Meek KM. X-ray scattering used to map the preferred collagen orientation in the human cornea and limbus. Structure 2004; 12: 249-256.

18 Daxer A, Fratzl P. Collagen fibril orientation in the human corneal stroma and its implications in keratoconus. Invest Ophthalmol Vis Sci 1997; 38: 121-129.

19 Radner W, Zehetmayer M, Skorpik C, Mallinger R. Altered organization of collagen in the apex of keratoconus corneas. Ophthal Res 1998; 30: 327-332.

20 Canon DJ, Foster CS. Collagen crosslinking in keratoconus. Invest Ophthalmol Vis Sci 1978; 17: 63-65.

21 Fullwood NJ, Tuft SJ, Malik NS, Meek KM, Ridgway AE, Harrison RJ. Synchrotron x-ray diffraction studies of keratoconus corneal stroma. Invest Ophthalmol Vis Sci 1992; 33: 1734-1741.

22 Rawe IM, Meek KM, Leonard DW, Takahashi T, Cintron C. Structure of corneal scar tissue: an X-ray diffraction study. Biophys J 1994; 67: 1743-1748.

23 Pettinelli DJ, Starr CE, Stark WJ. Late traumatic corneal wound dehiscence after penetrating keratoplasty. Arch Opthal 2005; 123: 853-856.

24 Calkins JL, Hochheimer BF, Stark WJ. Corneal wound healing: holographic stress-test analysis. Invest Ophthalmol Vis Sci 1981; 21: 322-334. 